\title{
KINETIC ENERGY OF THE SWINGING SEPARATOR DRIVEN BY A LINEAR ELECTRIC MOTOR
}

\section{Marat Nafikov}

Bashkir State Agrarian University,

Ufa, Russian Federation

\section{Ramzid Akhmarov}

Bashkir State Agrarian

University,

Ufa, Russian Federation

\section{Rustam Aipov}

Bashkir State Agrarian

University,

Ufa, Russian Federation

IIshat Akhmetyanov

Bashkir State Agrarian

University,

Ufa, Russian Federation

\author{
Salavat Akchurin \\ Bashkir State Agrarian \\ University, \\ Ufa, Russian Federation \\ IInur Zagirov \\ Bashkir State Agrarian \\ University, \\ Ufa, Russian Federation
}

Crossref

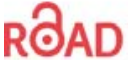

KOBSON

Key words: generalized coordinate, kinetic energy, linear asynchronous motor, mechanism, separator, swinging sieve

doi:10.5937/jaes18-25761

Cite article:

Nafikov M., Aipov R., Akchurin S., Akhmarov R., Akhmetyanov I., \& Zagirov, I. [2020]. Kinetic energy of the swinging separator driven by a linear electric motor. Journal of Applied Engineering Science, 18(3) $378-386$.

Online aceess of full paper is available at: www.engineeringscience.rs/browse-issues 


\title{
KINETIC ENERGY OF THE SWINGING SEPARATOR DRIVEN BY A LINEAR ELECTRIC MOTOR
}

\author{
Marat Nafikov*, Rustam Aipov, Salavat Akchurin, Ramzid Akhmarov, Ilshat Akhmetyanov, IInur Zagirov \\ Bashkir State Agrarian University, Ufa, Russian Federation
}

The goal of the given study is to develop a gearless mechanism driven by a linear electric motor that can reproduce and adjust sieve oscillation modes of large amplitude (more than $0.01 \mathrm{~m}$ ) and low frequency (less than 10-12 rad/s). The authors developed a test sample of a swinging separator driven by a linear electric motor and its kinematic scheme. There is a design scheme used to determine motion geometric and kinematic characteristics of the drive mechanism units as a function of the generalized coordinate $h$ - the stroke of a rotor relative to a stator in the linear electric motor $\partial / \partial t$. The paper provides kinetic energy expressions that are convenient for further development of a mathematical model for the mechanism movement based on Lagrange equations of the second kind. Most mechani$\mathrm{cal}$ and technical systems are studied with Lagrange equations being the scientific basis for the analysis and synthesis of a wide variety of machines and devices. This paper relies on the Lagrange equations to give a mathematical description of the sieves' movement in a grain cleaner. The paper presents kinetic energy expressions for the studied mechanism that are convenient for further development of a mathematical model of its motion based on Lagrange equations of the second kind. The results of the performed analytical studies can be used not only in the design of drive mechanisms for swinging sieves, but also in the design, upgrade and optimization of different industrial and agricultural machines driven by linear asynchronous motors.

Key words: generalized coordinate, kinetic energy, linear asynchronous motor, mechanism, separator, swinging sieve

\section{INTRODUCTION}

Current economic and technological requirements of grain production require highly adaptive resource-and energy-saving cleaning facilities [1, 10-12].

Grain mixtures are commonly separated with swinging sieves $[4,5]$. This cleaning scheme is used in well-known grain cleaners Westrup (Denmark), Alvan Blanch, Petkus (Germany), Lithuania (Lithuania), Taiho, ZYY (China), MGS (Russia), etc. In the drives of the executive units of known devices, electric rotation motors are used. Wide use of these cleaning schemes is explained by their easy technological process, the simple device design, the convenient layout of the machine, and its low energy consumption [5-8, 35].

Swinging sieves are widely used for separating grain mixtures $[1,2]$. Traditionally, swinging sieves are driven by asynchronous electric motors in combination with inertial vibrators [5].

The widespread use of such machines is explained by the simplicity of the cleaning process carried out on a flat sieve, the simplicity of the machine's design, and its convenient layout. These separators are less energy-consuming compared to air cleaning systems and Trier units $[4,5]$. Such a drive with a frequency-controlled asynchronous rotation motor makes it possible to change the frequency of vibrations of the sieves quite simply, continuously and without stopping the machine [5-8]. However, in traditionally used devices the vibration amplitude can be changed by manual replacement of the imbalance mass (stepwise) after the separator shutoff [6].
It's very difficult to adjust the angle of the sieves without stopping the grain cleaner.

Changing the design of grain cleaners driven by an electric rotation motor to address the noted shortcomings results in a heavier weight of the machine due to the need to ensure its strength. The cleaning device turns out to be complex, bulky and expensive.

The grain material to be separated has different impurities. There can be up to $15 \%$ of foreign matter [9]. Currently, each type of impurity can be separated on a specific type of machine $[4,7]$.

Modern economic and technological requirements of grain production require to create highly adaptive resource-and energy-saving technical means of cleaning [6]. Analysis of the technical literature and experience in creating grain cleaners with flat sieves shows that there is insufficient data to solve problems to increase their adaptability and efficiency while reducing the specific metal content and energy intensity of the equipment.

A working hypothesis is that the use of a linear asynchronous electric motor in the drive mechanism of a swinging separator will provide higher technical and economic characteristics of the device being developed [7-9]. This assumption is based on the following.

Grain mixtures separation is the most favorable when the oscillating movement of the executive link is of lower frequency (less than 2-3 Hz) and larger amplitudes (more than $0.01 \mathrm{~m}$ ) [1, 10-12]. A similar and easily adjustable oscillating process can be implemented directly from the rotor of a linear motor [7]. This simplifies the design and 
reduces the specific metal content of the developed machine as there is no need for a reducer and converting the rotation movement into a translational one.

Linear motors have a simple and multi-option design. They are easy to install, have a wide range of modes, and are relatively inexpensive $[13,14]$. They provide higher mixing intensity of the separated material's layers and more passing particles on the surface of the sieve to pass through its gaps for a short time [13].

All these positive aspects of grain cleaning can be realized by using a linear asynchronous motor in the drive of the swinging separator.

The purpose of this study is to develop a more advanced mechanism of the swinging separator by using a direct gearless drive of a linear electric motor. The motion of the moving part of the latter (the rotor) is set by a switch-an electronic control unit. The electronic control unit provides the necessary stator motion law of the linear motor. It must ensure the required rotor kinematics and create the necessary force to enable the operation of the drive mechanism. To create, configure and control such an electronic device is an engineering challenge, solved by developing a mathematical model of the movement of the machine drive. Such a mathematical model can be created on the basis of Lagrange equations of the second kind.

The subsequent analytical study involves modeling the movement of the mechanism being developed on the basis of Lagrange equations of the second kind.

To achieve this goal it is necessary to solve the following specific research tasks:

1. Select the kinematic scheme of the sieve drive mechanism.

2. Determine its rational geometric and kinematic parameters, while taking as a generalized coordinate the stroke of the rotor of a linear motor relative to its stator.

3. Determine the kinetic energy of the swinging separator as a function of the selected generalized coordinate and generalized speed.

\section{MATERIALS AND METHODS}

Analytical research methods are used to develop a mathematical model of the mechanism using Lagrange equations of the second kind [8].

Lagrange equations are the scientific basis for analyzing and synthesizing a wide variety of machines and devices. They are widely used not only in the design of transmission mechanisms [8, 11-13], but also in various fields of Engineering, Physics, and Economics. For example, the equations were used in research of underwater robots [14] (Canada), transmissions of machines and mechanisms [20-22], (USA, Russia, Belarus, Romania), machine mechanics [23] (Russia), Economics [24] (Ukraine), etc.
The most commonly used Lagrange equations (also known as equations of motion of a mechanical system in generalized coordinates) has the form

$\frac{d}{d t}\left(\frac{\partial T}{\partial \dot{q}_{j}}\right)-\frac{\partial T}{\partial q_{j}}=Q_{j}$

Where is the index (number) of the equation; $q_{j}-j$ - the generalized coordinate; $\dot{q}_{j}$ - the generalized velocity; $T$ - the kinetic energy of the system; $Q_{j}$ - the generalized force with respect to $j$ - the generalized coordinate. The kinetic energy of the system in the equations must be expressed in terms of generalized coordinates and generalized velocities.

\section{Design scheme}

The subject of these specific studies was a test sample of the mechanical sieve drive mechanism based on a cylindrical linear asynchronous electric motor, designed and manufactured at the Bashkir State Agrarian University (BSAU) [18]. Fig. 1 shows its kinematic design scheme.

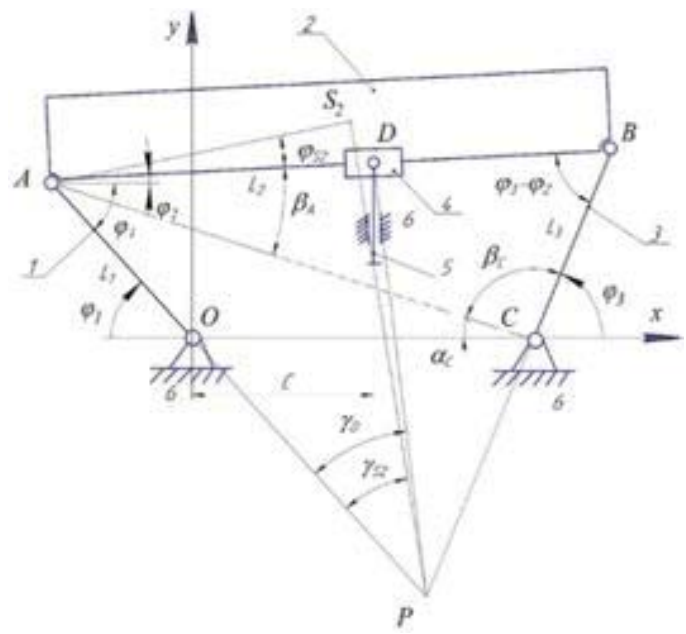

Figure 1: The drive mechanism diagram of the swinging separator operated by a linear electric motor

The mechanism consists of five movable units and a frame: 1-rocker arm; 2-connecting rod, made as one unit with the motor rotor; 3-rocker arm; 4-slider, being one unit with the engine stator; 5-rod. Rocker arms 1 and 3 make rotary movement about fixed axes $\mathrm{O}$ and $\mathrm{C}$ respectively; connecting rod 2 moves plane-parallel; slider 4 also moves plane-parallel. It rotates together with rod 2 and moves against it translatory; stator 4 is fixed on the translatory moving rod $5 ; 6$ - stationary rack (mechanism frame). The mechanism of the swinging separator is driven by a linear motor in accordance with the law set by the switch (electronic control unit).

\section{Geometric dimensions of the mechanism and the law of motion of the leading unit}

The geometric dimensions of the flat five-unit mechanism with one degree of freedom shown in Fig. 2, defined by preliminary calculations, are equal to: $O A=L_{1}=0.56 \mathrm{~m}$; $A B=L_{2}=1 \mathrm{~m} ; B C=L_{3}=0.59 \mathrm{~m} ; O C=0.28 \mathrm{~m} ; c=0.14 \mathrm{~m}[25]$. 


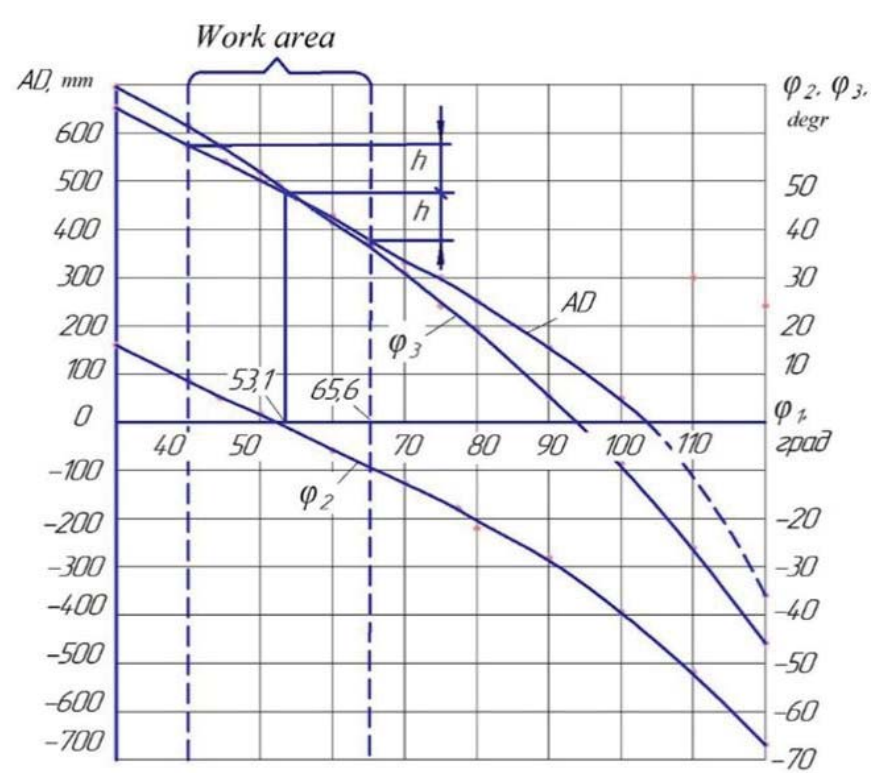

Figure 2: Dependence of the mechanism geometric parameters on unit 1 positions

The gravity center position of the swinging sieve $S_{2}$ is set by the parameters $A S_{2}=0.5 \mathrm{~m}, \varphi_{S 2}=20^{\circ}$ specified in the diagram.

The intersection point $P$ of the $O A$ and $B C$ lines is the instantaneous center of the connecting rod 2 . The angular coordinates of the units $\varphi_{1}, \varphi_{2}, \varphi_{3}$ and the positive directions of their reference are shown in Fig. 1, the instantaneous center of unit 2 is indicated in the diagram as $P$.

The oscillation frequency and stroke of the linear motor rotor relative to its stator are provided by setting up the switch - the electronic control unit of the machine. The switch sets the harmonic oscillatory law of the motor rotor motion in accordance with the dependence

$h=a \times \sin (k t)$

where $a$ is the amplitude of the oscillatory motion of the rotor; $k$ is the frequency of its oscillations.

In subsequent calculations, the values of $a=0.1 \mathrm{~m}$, $k=12 \mathrm{rad} / \mathrm{s}$ were taken.

In our research, we are primarily interested in the dependence of the kinematic parameters of the lever mechanism on the generalized coordinate and the generalized speed.

\section{RESULTS}

\section{Calculation of geometric parameters of the mechanism}

Using the above given dimensions of the mechanism, we will determine the geometric parameters that define the positions of its moving parts. In further studies of the mechanism it is planned to develop a mathematical model based on Lagrange equations of the second kind [15$17,26]$. In our case, when the mechanism is driven by a linear motor, the output of its stator is correctly selected as the generalized coordinate. Therefore, geometric parameters defining the position of the drive mechanism units were used as a function of angle $\varphi_{1}$ position of the rocker arm 1, as well as the rotor stroke $h$ relative to the stator of the linear motor depending on the generalized coordinate.

Using the calculation scheme in Fig. 2, we get the following ratios.

$\underline{\Delta O A C} . \quad \alpha_{C}=\operatorname{arctg}\left(\frac{L_{1} \cdot \sin \varphi_{1}}{O C+L_{1} \cdot \cos \varphi_{1}}\right) A C=L_{1} \frac{\sin \varphi_{1}}{\sin \alpha_{C}}$

$\underline{\Delta A B C} . \beta_{C}=\arccos \left(\frac{L_{3}^{2}+A C^{2}-L_{2}^{2}}{2 A C \cdot L_{3}}\right) \beta_{A}=\arccos \left(\frac{L_{2}^{2}+A C^{2}-L_{3}^{3}}{2 A C \cdot L_{2}}\right)$,

$\varphi_{2}=\beta_{A}-\alpha_{C}, \varphi_{3}=\pi-\beta_{C}-\alpha_{C}$.

$\underline{\triangle A B P} . A P=L_{2} \frac{\sin \left(\varphi_{3}-\varphi_{2}\right)}{\sin \left(\varphi_{1}+\varphi_{3}\right)} B P=L_{2} \frac{\sin \left(\varphi_{1}+\varphi_{2}\right)}{\sin \left(\varphi_{1}+\varphi_{3}\right)}$.

$\underline{\Delta A D P} . \quad A D=\frac{L_{1} \cdot \cos \varphi_{1}+c}{\cos \varphi_{2}}, \quad \gamma_{D}=\arcsin \left(\frac{A D}{D P} \sin \left(\varphi_{1}+\varphi_{2}\right)\right)$

$\boldsymbol{A A P S}_{2} \cdot P S_{2}=\sqrt{A P^{2}+A S_{2}^{2}-2 \cdot A P \cdot A S_{2} \cdot \cos \left(\varphi_{1}+\varphi_{2}+\varphi_{S 2}\right)}$,

$\gamma_{S 2}=\arcsin \left(\frac{A S_{2}}{P S_{2}} \sin \left(\varphi_{1}+\varphi_{2}+\varphi_{S 2}\right)\right)$.

The ordinate of a point $D$ belonging to units 4 and 5 is equal to

$y_{D}=L_{1} \cdot \sin \left(\varphi_{1}\right)+A D \cdot \sin \left(\varphi_{2}\right)$.

The results of calculations for dependencies $(3,4)$ are summarized in table 1.

As a result of the calculations Fig. 2 presents graphs of the dependence of the swing angles of units 2 and 3 , as well as the position of the hinge $D$ on the connecting rod ABrelative to the angular coordinate $\varphi_{1}$ of the rocker arm $O A$.

Analyzing the results of calculations, we select the operating range of the test mechanism. At values of the stator stroke relative to the rotor $h= \pm 0.1 \mathrm{~m}$, the oscillation angle of unit 1 changes within the limits $40^{\circ} \leq \varphi \leq 65.6^{\circ}$, which provides favorable pressure angles in the hinge $A$. The pressure angle at the hinge $B$ has a satisfactory value as well. In the selected operating range, the oscillation angle of the separator sieves $\varphi_{2}$ varies from 9.23 to $-9.23^{\circ}$, that corresponds to the literature recommendations [3, etc.]. These sources show that the rational values of the sieve inclination in traditional grain cleaners depend on the type of grain and are about 6-8 degrees. Therefore, the designed machine allows you to sift almost all types of seeds of agricultural crops.

In the mechanism, the amplitude of the linear oscillatory motion of the executive second unit corresponds to the rotor $h$ stroke of the electric motor.

The calculations of the geometric parameters of the mechanism confirm the correct choice of the scheme and the above-mentioned dimensions of the sieve drive mechanism.

In our research, we are primarily interested in the dependence of the geometric parameters of the mechanism not on the angle, $\varphi_{1}$ but on the generalized coordinate $h$. 
Table 1: Geometric parameters of the investigated mechanism

\begin{tabular}{|c|c|c|c|c|c|c|c|c|c|c|}
\hline No. & $\varphi_{1}$ degr & $\varphi_{2}$, degr & $\varphi_{3}$, degr & $P S_{2}, \mathrm{~mm}$ & $Y_{D,} m m$ & $A P, \mathrm{~mm}$ & $B P, \mathrm{~mm}$ & $A D, \mathrm{~mm}$ & $h, \mathrm{~mm}$ & Notes \\
\hline 1 & 30 & 16.05 & 70.59 & 774.79 & 459.80 & 828.67 & 732.43 & 650.32 & - & - \\
\hline 2 & 40 & 9.23 & 61.88 & 788.58 & 452.43 & 812.36 & 773.92 & 576.45 & -100.00 & \multirow{8}{*}{$\begin{array}{l}\text { The } \\
\text { operating } \\
\text { range }\end{array}$} \\
\hline 3 & 45 & 5.76 & 57.27 & 792.30 & 450.05 & 801.06 & 792.62 & 538.70 & -62.25 & \\
\hline 4 & 50 & 2.24 & 52.49 & 793.64 & 448.50 & 787.48 & 809.69 & 500.34 & -23.89 & \\
\hline 5 & 53.073 & 0.0370 & 49.452 & 793.21 & 447.98 & 777.95 & 819.29 & 476.45 & 0 & \\
\hline 6 & 55 & -1.36 & 47.51 & 792.44 & 447.81 & 771.50 & 824.94 & 461.33 & 15.12 & \\
\hline 7 & 60 & -5.03 & 42.23 & 788.53 & 448.02 & 753.02 & 838.22 & 421.62 & 54.83 & \\
\hline 8 & 65 & -8.80 & 36.94 & 781.81 & 449.23 & 732.00 & 849.38 & 381.15 & 95.30 & \\
\hline 9 & 65.574 & -9.24 & 36.306 & 780.86 & 449.84 & 729.42 & 850.52 & 376.45 & 100.00 & \\
\hline 10 & 70 & -12.69 & 31.31 & 772.19 & 451.60 & 708.40 & 858.33 & 339.83 & - & - \\
\hline 11 & 75 & -16.71 & 25.43 & 759.60 & 455.37 & 682.26 & 865.01 & 297.50 & - & - \\
\hline 12 & 80 & -20.90 & 19.27 & 744.04 & 460.89 & 653.63 & 869.40 & 253.96 & - & - \\
\hline 13 & 90 & -29.91 & 5.97 & 704.14 & 479.46 & 589.27 & 871.53 & 161.51 & - & - \\
\hline 14 & 100 & -40.04 & -8.96 & 653.15 & 515.56 & 516.39 & 865.79 & 55.85 & - & - \\
\hline 15 & 110 & -51.82 & -26.13 & 592.30 & 591.75 & 436.00 & 854.63 & -83.36 & - & - \\
\hline 16 & 120 & -66.15 & -46.73 & 523.40 & 801.60 & 347.10 & 843.21 & -346.2 & - & - \\
\hline
\end{tabular}

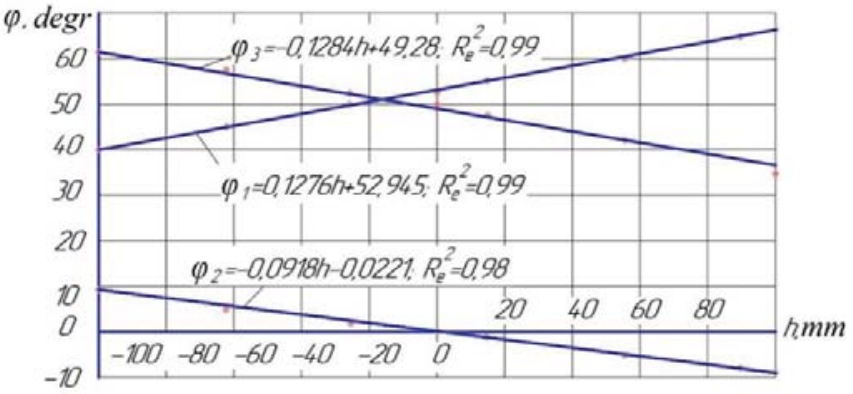

Figure 3: Mechanism unit position angle dependencies on the rotor $h$ stroke of the linear motor

The desired dependence of the oscillation angles of the units on the parameter $h$ is illustrated by the graphs in Fig. 3. The graphs are based on the calculation results shown in the Table 1.

The graphs show that oscillation angles $\varphi_{1}, \varphi_{2}$, and $\varphi_{3}$ of the units within the mechanism performance range change linearly. The dependencies can be expressed by equations:

$$
\varphi_{1}=2.227 \cdot h+0.924, \quad \varphi_{2}=1.613 \cdot h+0.0003,
$$$$
\varphi_{3}=2.241 \cdot h+0.860
$$

In equations (5), that will later be called empirical, the stroke $h$ is in meters, the angles $\varphi$ are in radians.

Similarly, according to the results shown in table 1 , the linear geometric dimensions of the mechanism within its operating range are determined:

$P S_{2}=-0.0484 \cdot h+0.789 y_{D}=0.3 h^{2}-0.0142 \cdot h+0.448$ (6)
The values $h, P S_{2}, \quad y_{D}$ are given in meters.

The values of the geometric parameters of the mechanism calculated from the dependencies $(5,6)$ do not differ from the data in Table 1.

Variable $P S_{2}$ - the distance to the instantaneous center of unit 2 within the operating range of the mechanism changes slightly (less than $1 \%$ ).

\section{Determination of linear and angular velocities}

Based on the empirical equations $(5,6)$, the linear and angular velocities of the mechanism are found as functions of the generalized speed, time, and generalized coordinate.

The dependence of unit speeds on the generalized velocity $h$ is obtained by differentiating the empirical relations $(5,6)$ :

$\omega_{1}=2.227 \cdot h \omega_{2}=1.613 \cdot h \omega=2.241 \cdot h$

$$
\begin{aligned}
& v_{S 2}=w_{2} \cdot P S_{2}=-1.613 \cdot h \cdot\left(-0.0484 \cdot \frac{\sqrt{a^{2}+k^{2}-h^{2}}}{k}+0.789\right) \\
& v_{S 4}=v_{5}=y_{D}=0.6 \cdot h \cdot h-0.0142 \cdot h= \\
& =h \cdot\left(0.6 \cdot \frac{\sqrt{a^{2}+k^{2}-h^{2}}}{k} \cdot h-0.0142\right)
\end{aligned}
$$

Here, the generalized coordinate is equal to,

$h=a \times \sin (k t)$

the generalized velocity

$\dot{h}=a k \cdot \cos (k t)$.

Speed equations (7) are copied as a time function:

$$
\begin{aligned}
& \omega_{1}=2.227 \cdot a k \cdot \cos (k t), \omega_{2}=1.613 \cdot a k \cdot \cos (k t), \omega_{3}=2.241 \cdot a k \cdot \cos (k t) \\
& v_{S 2}=0.0390 \cdot a^{2} \cdot k \cdot \sin (2 k \cdot t)-0.0142 \cdot a \cdot k \cdot \cos (k t) \\
& v_{S 4}=v_{S 5}=0.3 a^{2} \cdot k \cdot \sin (2 k \cdot t)-0.0142 \cdot k \cdot \cos (k t)
\end{aligned}
$$


The mechanism unit velocities are expressed as a function of the generalized coordinate $h$ :

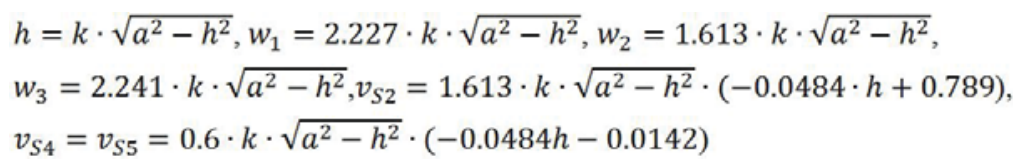

Equations (7-9) to find the linear and angular velocities of mechanism units are obtained by differentiating the empirical geometric relations (2-5). The resulting kinematic equations are simple due to the fact that the mechanism unit velocities are expressed in terms of a generalized coordinate and a generalized speed. However, it is necessary to assess their accuracy.

To check the obtained expressions (7-9) for angular velocities to be correct and accurate, these parameters are also determined in the traditional way. The latter involves building speed plans and drawing up gear ratios [16,17]. We use the calculation schemes in Fig 4a, 4b.

Velocities of the mechanism points are associated with vector equations:

$\bar{v}_{D 2}=\bar{v}_{A}+\bar{v}_{D 2 A}, \quad \bar{v}_{D 2}=\bar{v}_{D}+\bar{v}_{D 2 D}$

Equations are solved (10). By designing the velocity vectors on the axis $O x$, we get:

$v_{D 2} \cdot \sin \left(\varphi_{1}+\gamma_{D}\right)=v_{A} \cdot \sin \left(\varphi_{1}\right)+v_{D 2 A} \cdot \sin \left(\varphi_{2}\right)=$
$=0+v_{D 2 D} \cdot \cos \left(\varphi_{2}\right)$

from which

$v_{D 2}=v_{D 2 D} \frac{\cos \left(\varphi_{2}\right)}{\sin \left(\varphi_{1}+\gamma_{D}\right)}$

According to the diagram in Fig. 4, a we connect the speeds of the unit 2 points:

$\frac{v_{A}}{A P}=\frac{v_{D 2}}{D P}=\frac{v_{B}}{B P}=\frac{v_{S 2}}{P S_{2}}=\omega_{2}$

We take into account that $v_{A}=\omega_{1} \cdot L_{1}$ and $v_{B}=\omega_{3} \cdot L_{3}$. There are expressions from (13) for the transfer relations between the angular velocities:

$i_{21}=\frac{\omega_{2}}{\omega_{1}}=\frac{L_{1}}{A P}, \quad i_{32}=\frac{\omega_{3}}{\omega_{2}}=\frac{B P}{L_{3}}, \quad i_{31}=\frac{\omega_{3}}{\omega_{1}}=i_{21} \cdot i_{32}$.

The values found according to the transfer relations (12) and (14) angular velocities of the units are shown in table 2 , where they are indicated $\omega_{1 \pi}, \omega_{2 \pi}, \omega_{3 \pi}$. The parameter values $a=0,1 \mathrm{~m}$ and $k=12 \mathrm{rad} / \mathrm{s}$ are used for calculations. Table 2 presents the values of angular velocities to be compared calculated from empirical dependencies $(6,8)$ as well.

Speed ratio (7-9) are expressed via the generalized speed and generalized coordinate. It enables to express the kinetic energy of the mechanism as inner function $h$ and $\dot{h}$. Imprecision of angular velocities by empirical ratios (6-8) from the angular velocities found in the traditional way does not exceed $2-3 \%$. Calculations based on the obtained empirical equations (7-9) are simpler and more convenient.

\section{Determination of the kinetic energy of the mechanism}

Taking into account the motion nature of the mechanism units, an expression for calculating its kinetic energy is compiled:

$T=T_{1}+T_{2}+T_{3}+T_{4}+T_{5}=$

$=\frac{1}{2} I_{10} \cdot \omega_{1}^{2}+\frac{1}{2} m_{2} \cdot v_{S 2}^{2}+\frac{1}{2} I_{2 S} \cdot \omega_{2}^{2}+\frac{1}{2} I_{3 C} \cdot \omega_{3}^{2}+\frac{1}{2} m_{4} \cdot v_{S 2}^{2}+$

$+\frac{1}{2} I_{4 S} \cdot \omega_{4}^{2}+\frac{1}{2} m_{5} \cdot v_{S 5}^{2}$

The calculation results of the kinetic energies of the units and the entire mechanism are shown in Table 3.

Calculations have shown that the kinetic energy of the mechanism is mostly affected by the kinetic energy $T_{2}$ of the executive unit and the terms $T_{1}, T_{3}, T_{4}$ and $T_{5}$ in expression (15) are negligible. Their total kinetic energy does not exceed $1 \%$ of the total kinetic energy of the entire mechanism.

$$
\begin{aligned}
& T(h)-120.46-14.31 \cdot h-12039 \cdot h^{2}+ \\
& +1430 \cdot h^{3}-36.27 \cdot h^{4} \\
& T_{2}(h)=83.74 \cdot h^{2}-0.0021 \cdot h^{4}-0.828 \cdot h^{2} \sqrt{1.44-h^{2}}
\end{aligned}
$$
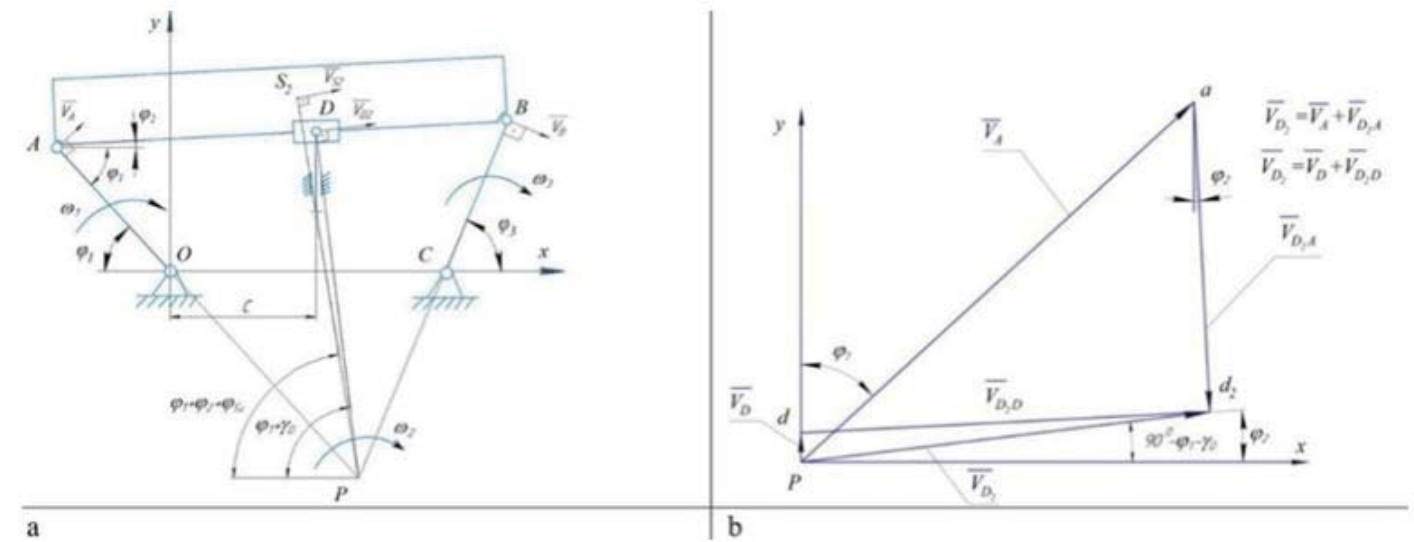

Fig. 4. To find transfer relations for the angular velocities of units: $a$ - kinematic scheme of the drive mechanism; $b$ - speed plan 
Table 2: Values of angular velocities of the mechanism units depending on the generalized coordinate $h$ and the generalized speedu $v_{D 2 D}$ found by different ways

\begin{tabular}{|c|c|c|c|c|c|c|c|c|c|}
\hline No. & $t, s$ & $h, m$ & $v_{D 2 D}, \mathrm{~m} / \mathrm{s}$ & $\omega_{1 \pi}, \mathrm{rad} / \mathrm{s}$ & $\omega_{2 \pi}, \mathrm{rad} / \mathrm{s}$ & $\omega_{3 \pi}, \mathrm{rad} / \mathrm{s}$ & $\omega_{1}, \mathrm{rad} / \mathrm{s}$ & $\omega_{2}, \mathrm{rad} / \mathrm{s}$ & $\omega_{3^{\prime}}, \mathrm{rad} / \mathrm{s}$ \\
\hline 0 & 0 & 0 & 1.200 & 2.690 & -1.928 & -2.687 & 2.672 & -1.936 & -2.689 \\
\hline 1 & 0.044 & 0.050 & 1.039 & 2.262 & -1.693 & -2.377 & 2.314 & -1.676 & -2.329 \\
\hline 2 & 0.087 & 0.087 & 0.600 & 1.273 & -0.992 & -1.390 & 1.336 & -0.968 & -1.345 \\
\hline 3 & 0.131 & 0.100 & 0 & 0 & 0 & 0 & 0 & 0 & 0 \\
\hline 4 & 0.175 & 0.087 & -0.600 & -1.273 & 0.992 & 1.390 & -1.336 & 0.968 & 1.345 \\
\hline 5 & 0.218 & 0.050 & -1.039 & -2.262 & 1.693 & 2.377 & -2.314 & 1.676 & 2.329 \\
\hline 6 & 0.262 & 0 & -1.200 & -2.690 & 1.928 & 2.687 & -2.672 & 1.936 & 2.689 \\
\hline 7 & 0.305 & -0.050 & -1.039 & -2.386 & 1.686 & 2.268 & -2.314 & 1.676 & 2.329 \\
\hline 8 & 0.349 & -0.087 & -0.600 & -1.397 & 1.007 & 1.281 & -1.336 & 0.968 & 1.345 \\
\hline 9 & 0.393 & -0.100 & 0 & 0 & 0 & 0 & 0 & 0 & 0 \\
\hline 10 & 0.436 & -0.087 & 0.600 & 1.397 & -1.007 & -1.281 & 1.336 & -0.968 & -1.345 \\
\hline 11 & 0.480 & -0.050 & 1.039 & 2.386 & -1.686 & -2.268 & 2.314 & -1.676 & -2.329 \\
\hline 12 & 0.524 & 0 & 1.200 & 2.690 & -1.928 & -2.687 & 2.672 & -1.936 & -2.689 \\
\hline
\end{tabular}

Table 3: Dependences of the kinetic energies of the units and the mechanism as a whole on the generalized coordinate and the generalized velocity

\begin{tabular}{|c|c|c|c|c|c|c|c|c|c|c|}
\hline No. & $h, \mathrm{~m}$ & $v_{D 2 D}, \mathrm{~m} / \mathrm{s}$ & $T_{1}, \mathrm{~J}$ & $T_{2 \Pi}, \mathrm{J}$ & $T_{2 B}, \mathrm{~J}$ & $T_{3}, \mathrm{~J}$ & $T_{4 \Pi}, \mathrm{J}$ & $T_{4 B}, \mathrm{~J}$ & $T_{5}, \mathrm{~J}$ & $T, \mathrm{~J}$ \\
\hline 1 & 0 & 1.200 & 0.014 & 116.58 & 3.747 & 0.018 & 0.003 & 0.187 & 0.001 & 120.6 \\
\hline 2 & 0.050 & 1.039 & 0.011 & 86.896 & 2.810 & 0.014 & 0.003 & 0.140 & 0.001 & 89.86 \\
\hline 3 & 0.087 & 0.600 & 0.006 & 28.835 & 0.937 & 0.005 & 0.005 & 0.047 & 0.003 & 29.83 \\
\hline 4 & 0.100 & 0 & 0 & 0 & 0 & 0 & 0 & 0 & 0 & 0 \\
\hline 5 & 0.087 & -0.600 & 0.006 & 28.835 & 0.937 & 0.005 & 0.005 & 0.047 & 0.003 & 29.83 \\
\hline 6 & 0.050 & -1.039 & 0.011 & 86.896 & 2.810 & 0.014 & 0.003 & 0.140 & 0.001 & 89.86 \\
\hline 7 & 0 & -1.200 & 0.014 & 116.58 & 3.747 & 0.018 & 0.003 & 0.187 & 0.001 & 120.6 \\
\hline 8 & -0.050 & -1.039 & 0.011 & 86.896 & 2.810 & 0.014 & 0.003 & 0.140 & 0.001 & 89.86 \\
\hline 9 & -0.087 & -0.600 & 0.006 & 28.835 & 0.937 & 0.005 & 0.005 & 0.047 & 0.003 & 29.83 \\
\hline 10 & -0.100 & 0 & 0 & 0 & 0 & 0 & 0 & 0 & 0 & 0 \\
\hline 11 & -0.087 & 0.600 & 0.006 & 28.835 & 0.937 & 0.005 & 0.005 & 0.047 & 0.003 & 29.83 \\
\hline 12 & -0.050 & 1.039 & 0.011 & 86.896 & 2.810 & 0.014 & 0.003 & 0.140 & 0.001 & 89.86 \\
\hline 13 & 0 & 1.200 & 0.014 & 116.58 & 3.747 & 0.018 & 0.003 & 0.187 & 0.001 & 120.6 \\
\hline
\end{tabular}

Hence, the dependencies of kinetic energies on the generalized coordinate $h$ and the generalized velocity $\dot{h}$ will have the following simpler form:

Using the expressions $(16,17)$, multiple calculations were performed and graphs of the mechanism's kinetic energy dependences on the generalized velocity and the generalized coordinate were constructed (Fig. 5).

The following values of the inertia characteristics of the units are used for calculations: $I_{1 A}=0.004 \mathrm{kgm}^{2}$, $I_{2 S}=2.0 \mathrm{kgm}^{2}, I_{3 \mathrm{C}}=0.005 \mathrm{kgm}^{2}, I_{4 \mathrm{~S}}=0.1 \mathrm{kgm}^{2}, \quad m_{2}=100 \mathrm{~kg}$, $m_{4}=20 \mathrm{~kg}, m_{5}=10 \mathrm{~kg}$.

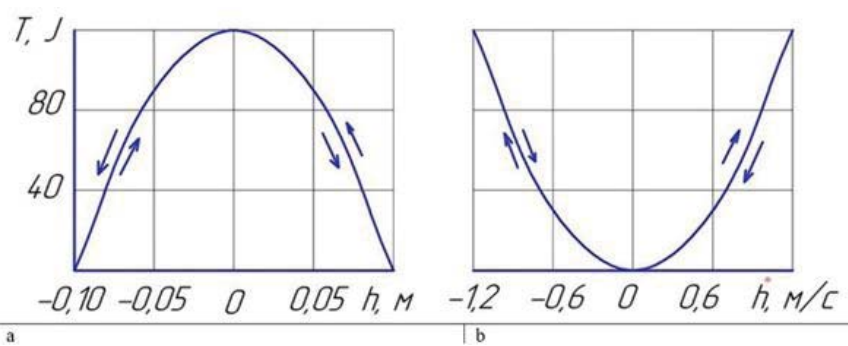

Figure 5: Dependences of the kinetic energy of the drive mechanism on: a - the generalized coordinate $h$, $b$ - the generalized speed $h=v_{D 2 D}$ 


\section{DISCUSSION}

Analysis of Russian and foreign technical literature and experience in creating grain cleaners with flat sieves show that there is insufficient information to meet the challenges of increasing their adaptability and efficiency. In order to increase the efficiency of swinging separators while reducing the specific metal content and energy intensity of the equipment, it is necessary to provide controlled oscillatory movement of the executive unit with low frequencies $(2-3 \mathrm{~Hz}$ ) and amplitudes larger than $0.01 \mathrm{~m}$ in comparison with traditionally used devices with periodic changes in the inclination angle of the sieves. All these positive aspects of grain cleaning are realized by using a linear asynchronous motor in the drive [1, 10-12]. Some difficulties in using linear electric motors in different fields are related to the need to develop an electronic control unit [30-34]. Such a device must provide the required motion law of the linear motor stator in accordance with the technological conditions of the machine [35-38].

In turn, the control unit creation is based on a mathematical model of the movement of the developed machine. In many cases, differential equations of motion of a system with one degree of freedom in generalized coordinates (Lagrange equations of the second kind) are used to model the motion of a machine [19].

The given research was aimed to develop a gearless grain cleaner that gave the following results.

The authors propose a kinematic scheme of the mechanism of a swinging separator with such an electric motor. A test sample of a grain cleaner was made. The dimension ratio of the mechanism with one degree of freedom is determined by preliminary calculations.

Since the mechanism is driven by a linear electric motor and the profile of the motor's thrust force is determined, the stroke of the $h$ rotor of a linear electric motor relative to its stator is chosen as a generalized coordinate in the Lagrange equation. The Lagrange equation (1) for the selected generalized coordinate takes the form

$\frac{d}{d t}\left(\frac{\partial T}{d \dot{h}}\right)-\frac{\partial T}{\partial h}=Q_{h}$.

In the equation (?), the kinetic energy of the mechanism had to be expressed in terms of a generalized coordinate $h$ and a generalized velocity $\dot{h}$.

The calculation scheme is shown in Fig. 1. Geometric calculations were performed in accordance with the scheme $(2,3)$. The positions of all five units of the mechanism (including the stroke $h$ of the rotor of a linear electric motor) were expressed in terms of the angular coordinate $\varphi_{1}$ of the crank 1 , which performs a rotational oscillatory movement around its axis $O$.

The calculations made it possible to choose a rational course of the leading unit $h= \pm 0,1 \mathrm{~m}$.

The choice of the stator stroke of the electric motor is illustrated by the graphs in Fig. 2. In the selected range of the generalized coordinate, the pressure angles in the hinges $A$ and $B$ have satisfactory values, and the oscillation angle $\varphi_{2}$ of the separator sieves varies in the rational range from 9.23 to $-9.23^{\circ}$, corresponding to the literature recommendations [3].

Based on the results of geometric calculations after statistical data processing, there are linear equations (5) that are more convenient for modeling dependences of the angular coordinates of the mechanism units on the generalized coordinate $h$. Similarly, according to the results shown in table 1 , the linear geometric dimensions of the mechanism (6) are determined depending on the parameter $h$.

The results of calculating the geometric parameters of the studied mechanism based on empirical dependencies $(5,6)$ do not differ from the values in table 1 .

Then, by differentiating the geometric dependencies $(5,6)$, equations $(7)$ for center-of-mass velocities of the mechanism units and their angular speeds relative to the generalized velocity are compiled $\dot{h}$. Similarly, dependences (8) of kinematic parameters of solids 1-5 on time and dependences (9) on the generalized coordinate are obtained $h$.

The accuracy of empirical equations (7-9) is confirmed by more precise methods of the theory of mechanisms and machines to find velocities of the drive mechanism units [20].

The discrepancy between angular velocity calculation results based on empirical ratios (7-9) and the values found by the traditional method does not exceed $2-3 \%$.

The expression (15) of the kinetic energy of the mechanism under study is obtained as the sum of the kinetic energies of all its five units. The results of calculations using the formula (15), shown in table 3 , showed that the kinetic energy of the mechanism is most affected by kinetic energy $T_{2}$. The kinetic energy of the remaining units 1, 3, 4 and 5 together make up no more than $1 \%$ of the kinetic energy of the entire mechanism. Therefore, we assume that the summand $T_{1}, T_{3}, T_{4}$ and $T_{5}$ in the expression (15) are negligible.

Accordingly, there are the expressions $(16,17)$ of the kinetic energy of the mechanism under study as a function of the generalized coordinate $h$ and the generalized velocityh $\dot{h}$, respectively. These expressions accept drawing up a necessary differential equation of partial derivatives (18) $\partial T / \partial h \partial T / \partial \dot{h}[35-38]$.

The conducted research on the development of a control mechanism for a grain cleaner driven by a linear electric motor is not complete.

After completing the mathematical model of the movement of the developed machine, it is planned to design and manufacture an electronic control device for a linear asynchronous electric motor. Such a device should provide a profile of the thrust force of the linear engine, which ensures the rational movement of the machine links in accordance with the established laws. 
The advantages of drives with linear asynchronous electric motors are the simplicity of the machine design, reduced weight and energy consumption, the ability to continuously control the frequency of the technological process, etc. Therefore, such drives can be more widely used not only for swinging sieves of grain cleaners, but also in a number of other industrial and agricultural machines.

\section{CONCLUSION}

The paper provides a kinematic scheme and a test sample of the swinging separator mechanism for dressing the grain mixture. As a result of using an asynchronous linear electric motor for its drive, there are more favorable conditions for the grain mixture cleaning in comparison with existing machines driven by rotation motors. The advantages of the developed mechanism are the possible non-stop regulation of oscillating plane-parallel movement of the sieves (amplitude, frequency and oscillation angles), fewer energy costs of the grain mixture separation, no need to apply energy-consuming, bulky drive components (reducer, a device converting rotational motion into linear motion, etc.).

The movement of the rotor of a linear electric motor relative to its stator is offered to be used as a generalized coordinate that determines the positions of the mechanism units. There are found linear and angular velocities of the lever mechanism units depending on the generalized coordinate $h$ and the generalized velocity $\dot{h}$. There are expressions of the kinetic energy of the mechanism in the form of equations $(16,17)$, being convenient to make partial derivatives $\partial T / \partial h$ and $\partial T / \partial \dot{h}$ that are included in the Lagrange equations.

The further research is to compile and analyze a mathematical model of the grain cleaner performance to develop an electronic control unit for a linear electric motor. The methods and results of the conducted analytical studies can be used not only in the design of drive mechanisms for swinging sieves, but also in the design, upgrade and optimization of different industrial and agricultural machines driven by linear asynchronous electric motors.

\section{REFERENCES}

1. Lapshin, I.P., Kasimov, N.I. (2002). Calculation and design of grain cleaners. Publishing Enterprise "Zauralie", Kurgan.

2. Bohnet, M. (2004). Mechanische Verfahrenstechnik. WILEY-VCH Vergal GmbH \& Co. Kga A, Weinheim.

3. Galkin, V.D., Koshurnikov, A.F., Khavyev, A.A., Khandrikov, V.A., Grubov, K.A. (2014). The vibroseparators and their use in the seed cleaning lines: textbook for universities. Publishing Centre "Prokrost", Perm.
4. Noorlandt, R., Drijkoningen, G., Dams, J., Jenneskens, R. (2015). A seismic vertical vibrator driven by linear synchronous motors. Geophysics, vol. 80, no. 2, 57-67.

5. Fedorenko, I.Ya. (2016). Vibration processes and devices in agriculture. Monograph. Editorial and Publishing department of the Altay State Agrarian University, Barnaul.

6. Bulat, P. V. (2014). About the detonationengine. American Journal of Applied Sciences, vol. 11, no. $8,1357-1364$

7. Linenko, A.V., Gabitov, I.I., Baynazarov, V.G., Tuktarov, M.F., Aipov, R.S., Akchurin, S.V., Kamalov, T.I., Badretdinov, I.D., Leontiev, D.S., Vokhmin, V.S. (2019). The mechatronic module "linear electric drive - sieve boot" intelligent control system of grain cleaner. Journal of the Balkan Tribological Association, vol. 25, no. 3, 708-717.

8. Michalczyk, J., Cieplok, G. (2016). Maximal amplitudes of vibrations of the suspended screens, during the transient resonance. Archives of Mining Sciences, vol. 61, no. 3, 537-552.

9. Liu, X.-P., Zhang, Y.-L., Yang, D. (2014). Finite element analysis of 5XF150/180 type grain cleaning machine. International Conference on Mechanism Science and Control Engineering (MSCE), p. 112118.

10. Khomenko, A.P., Kargapoltsev, S.K., Eliseev, A.V. (2018). Development of approaches to creation of active vibration control system in problems of the dynamics for granular media. International Conference on Engineering Vibration (ICoEV), Sofia, vol. 148, UNSP 11004

11. Zorawski, D., Dzikowska, W., Peszynski, K. (2014). Modelling of the vibrating dryer drive system. 20th International Conference on Engineering Mechanics (EM), Svratka, Czech Republic, p. 748-751.

12. Yarullin, R., Aipov, R., Gabitov, I., Linenko, A., Akchurin, S., Mudarisov, S., Khasanov, E., Rakhimov, Z., Masalimov, I. (2018). Adjustable driver of grain cleaning vibro-machine with vertical axis of eccentric masses rotation. Journal of Engineering and Applied Sciences, vol. 13, no. 88, 6398-6406.

13. Aipov, R.S., Yarullin, R.B., Gabitov, I.I., Mudarisov, S.G., Linenko, A.V., Farhshatov, M.N., Khasanov, E.R., Gabdrafikov, F.Z., Yukhin, G.P., Galiullin, R.R. (2018). Mechatronic system linear swing vibrating screen of a grain cleaner. Journal of Engineering and Applied Sciences, vol. 13, 6473-6477.

14. Kornev, A.S., Orobinskii, V.I., Sundeev, A.A. (2014). Higher efficiency of flat sieves operation in grain cleaners. Innovative Technologies and Technical Means for Agriculture, vol. 3, 84-89. 
15. Vulfson, I. (2015). Dynamics of cyclic machines. Series: Foundations of Engineering Mechanics, Vol. 19. Springer Verlag.

16. Aipov, R.S., Akchurin, S.V., Pugachev, V.V. (2018). Patent for an invention No. 2648755 RF "Vibration separator", Published 28.03.2018.

17. Nafikov, M., Gabitov, I., Aipov, R. (2019). Kinematic parameters of the swinging separator driven by a linear electric motor. Journal of the Balkan Tribological Association, vol. 25, no. 3, 832-844.

18. Aipov, R.S., Linenko, A.V., Tuktarov, M.F., Bainazarov, V.G. (2017). Analysis of pulsed operating mode of linear induction drive of grain cleaning. Collected papers: Actual issues of Mechanical Engineering (AIME 2017) Processing, p. 420-424.

19. Butenin, N.V., Lunts, J.L., Merkin, D.R. (2002). The course of theoretical mechanics: in 2 volumes. Doe, St. Petersburg.

20. Kurc, K., Szybicki, D. (2016). Determination of the dynamic parameters of underwater robots with tracked drives. Applied Mechanics and Materials, vol. 817, 130-139.

21. Samanuhut, P., Dogan, P. (2008). Dynamics Equations of Planetary Gear Sets for Shift Quality by Lagrange Method. Dynamic Systems and Control Conference, October 20-22, 2008, Ann Arbor, Michigan (USA), p. 353-360.

22. Yutao, L., Di, T. (2011). Dynamics modeling of planetary gear set considering meshing stiffness. Based on Bond Graph. Procedia Engineering, vol. 24, 850855.

23. Gorbatenko, N. N. (2017). Mathematical modeling of the process of switching stages in the planetary gearbox of a car. Tomsk State University Journal, vol. 4, 5-16.

24. Bataus, M., Maciac, A., Oprean, M., Vasiliu, N. (2011). Automotive clutch models for real time simulation. Proceedings of the Romanian academy. Series $A$, vol. 12, no. 2, 109-116.

25. Sarapulov, F., Sarapulov, S., Smolyanov, I. (2017). Research of thermal regimes of linear induction motor. 18th International Conference on Computational Problems of Electrical Engineering (CPEE), p. 1-4.

26. Algin, V.B. (2014). Dynamics of multi-mass systems of machines when changing states friction components and directions of power flows. Mechanics of machines, mechanisms and materials, vol. 4, 21-32.
27. Artobolevsky, I.I. (2011). Theory of mechanisms and machines. Publishing House Alliance, Moscow.

28. Erdman, A.G., Kota, S., Sandor, G.N. (2001). Mechanism Design: Analysis and Theory. Fourth Edition. Prentice Hall.

29. Mata, A., Torras, A., Garrillo, J., Juanco, F., Fernandez, A., Martinez, F. (2016). Fundamentals of Machine Theory and Mechanisms. Springer.

30. Gyriev, B.I., Kutusheva, L.S., Rusak, L.L. (2008). Theory of mechanisms and machine: Textbook, manual. Ufa State Aviation Technical University, Ufa.

31. Zagirnyak, M., Kalinov, A., Chumachova, A. (2013). Correction of operating condition of a variable-frequency electric drive with a non-linear and asymmetric induction motor. Collected papers: IEEE EuroCon 2013, p. 1033-1037.

32. Nahdi, T., Maga, D. (2018). Comparative study of frequency converters for doubly fed induction machines. Sustainability, vol. 10, no. 3, 594.

33. Mustafa, G.M., Gusev, S.I., Kuzikov, S.V., Chernov, I.S. (2018). A combined frequency converter for soft acceleration of induction electric drives with heavy starting conditions. Russian Electrical Engineering, vol. 89, no. 4, 282-286.

34. Rinkeviciene, R., Smilgevicius, A. (2007). Linear Induction Motor at Present Time. Electronics and Electrical Engineering, vol. 6, no. 78, 3-8.

35. Cirrincione, M., Pucci, M., Vitale, G., Sferlazza, A. (2010). Neural based MRAS sensorless techniques for high performance linear induction motor drives. Collected papers: IECON Proceedings (Industrial Electronics Conference), p. 918-926.

36. Sakamoto, Y., Kashiwagi, T., Hasegawa, H., Sasakawa, T., Fujii, N. (2011). Design considerations and experimental verification of a rail brake armature based on linear induction motor technology. IEEJ Transactions on Industry Applications, vol. 131, no. 1, 127-134.

37. He, Yu., Wang, Y.Sh., Lu, Q., Zhang, L., Liang, F. (2018). Design of single-sided linear induction motor for low-speed maglev vehicle in $160 \mathrm{~km} / \mathrm{h}$ and variable slip frequency control, Transport systems and technologies, vol. 4, no. 2, 120-128.

38. Tiunov, V.V. (2014). Electromagnetic fields, characteristics and practical structures of linear induction machines with a short operating body. Acta Technica CSAV (CeskoslovenskAkademieVed), vol. 59, no. 2, 115-134.

Paper submitted: 19.03.2020.

Paper accepted: 25.06.2020.

This is an open access article distributed under the CC BY 4.0 terms and conditions. 